\title{
Localized SiO emission triggered by the passage of the W51C supernova remnant shock
}

\section{G. Dumas*}

IRAM, Grenoble, F-38041, France

E-mail: gdumaseiram.fr

\section{S. Vaupré, C. Ceccarelli, P. Hily-Blant, G. Dubus}

UJF-Grenoble 1 / CNRS-INSU, IPAG UMR 5274, Grenoble, F-38041, France

\section{T. Montmerle}

IAP, France

\section{S. Gabici}

APC, AstroParticule et Cosmologie, Université Paris Diderot, CNRS, CEA, Observatoire de Paris, Sorbonne Paris, France

\begin{abstract}
The region toward W51C is a good example of interaction between a supernova remnant (SNR) and a molecular cloud. Large electron abundances have been reported toward the position W51CE located in this interaction region, and it was proposed that the enhanced ionization fraction was due to cosmic ray particles freshly accelerated by the SNR shock. Here we present Plateau de Bure Interferometer observations of the $\mathrm{H}^{13} \mathrm{CO}^{+}(1-0)$ and $\mathrm{DCO}^{+}(2-1)$ emission lines centered at position W51C-E. These observations confirm the previous scenario of cosmic-ray-induced ionization at this location and in addition, $\mathrm{SiO}(2-1)$ emission has been successfully mapped in the close vicinity of W51C-E, with a spatial resolution of 7'. The morphology and kinematics of the $\mathrm{SiO}$ emission which strongly suggest that this emission was produced by the passage of the SNR primary shock. Put in conjunction with the enhanced ionization fraction in this region, we give a consistent picture of this region, in which the W51C-E position is located downstream of the SNR primary shock, where a large reservoir of freshly accelerated particles is available.
\end{abstract}

Cosmic Rays and the InterStellar Medium - CRISM 2014,

24-27 June 2014

Montpellier, France

\footnotetext{
* Speaker.
} 


\section{Introduction}

Cosmic rays (CRs) permeate our Galaxy, playing a major role in setting the prevalent physical condition, notably because they are responsible for the ionization of the dense part of the molecular clouds, where UV and X-ray photon cannot penetrate. In these regions CRs are the major driver of the chemistry and of the physical parameters such as gas temperature and density. They also play an important role in the destruction of dust particles. Low-energy CRs $(\lesssim 1 \mathrm{Gev})$ can be probed by the enhancement of the ionization they cause in molecular gas [1].

Galactic CRs are thought to originate mostly from the shock of supernovae remnants (SNRs). Therefore, SNRs interacting with surrounding molecular clouds are promising sites for studying both the properties of freshly accelerated CRs and their feedback on their environment. In this context, the SNR W51C is an ideal target.

\section{The W51 complex}

The SNR W51C is situated in the W51 complex which consists of two star forming regions W51A and W51B in addition to the SNR (Fig. 1).

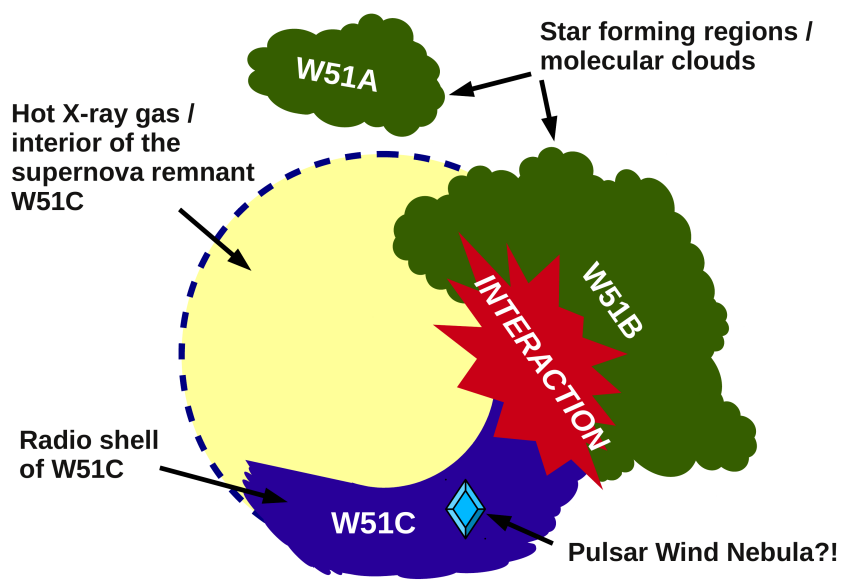

Figure 1: Illustration of the W51C region: W51A and W51B are two molecular clouds, hosting star formation, W51C is the supernova remnant, interaction with W51B. Image taken from J. Krause PhD thesis, 2012

It is situated $\sim 5^{\circ}$ away from the galactic plane at a distance of $\sim 5.5 \mathrm{kpc}$ [2]. The age of the SNR W51C is estimated to be about $\sim 3 \times 10^{4} \mathrm{yr}[3]$ and some observations provide evidence that the SNR is interacting with the nearby molecular cloud W51B. Figure 2 presents the environment of W51C. In particular a bright and extended GeV-TeV gamma-ray source is detected toward W51C by [4], [5] and [6], as shown in Fig. 2, left. These authors concluded that such emission comes from the interaction between the SNR and the nearby molecular cloud. The thermal X-ray emission, tracing the SNR primary shock extends northwest beyond the molecular cloud (Fig. 2, right), hinting that the SNR is situated behind the W51B cloud complex [3]. In addition, OH masers, high velocity $\mathrm{HI}$ cloud and molecular emission are coincidently observed in the overlapping regions of the W51C and W51B components, suggesting that they are associated with the SNR shock. 

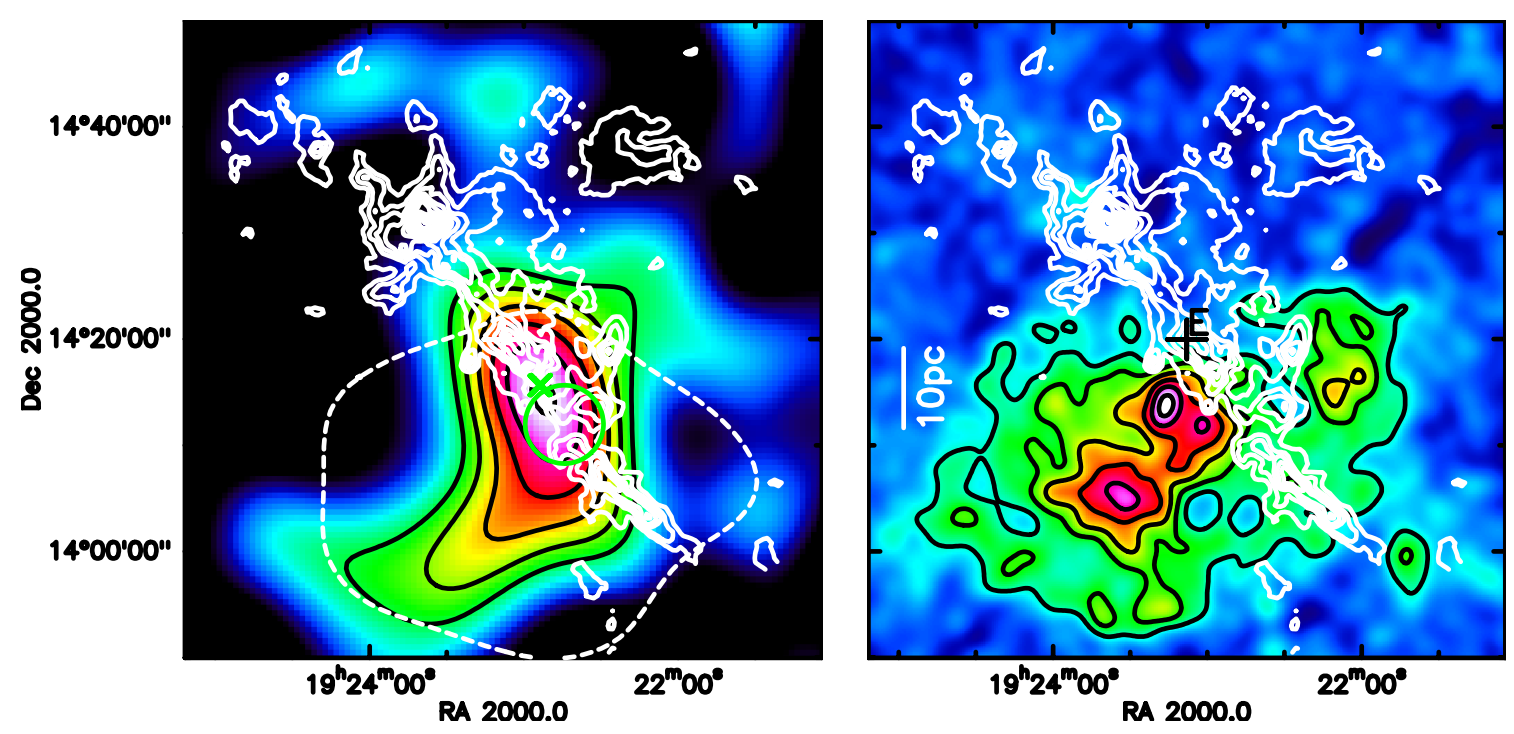

Figure 2: High energy environment of W51C. In both panels the black cross locates the phase center of our mm observations and white contours is showing the ${ }^{13} \mathrm{CO}(1-0)$ integrated intensity [7]. Left: MAGIC gamma-ray emission map from 300 tolo00 GeV [6]. The dashed contour marks the 260 counts deg $^{-2}$ measured by the Fermi/LAT in the 2-10 GeV energy range. Right: Rosat X-ray map of SNR W51C [3]

In dense environment, measurement of the abundance ratio $\mathrm{DCO}^{+} / \mathrm{HCO}^{+}$is a good probe of the ionization rate [8]. Therefore, in order to study the CR ionization rate in the interaction region between W51C and the molecular cloud, we started a mm observational campaign with the IRAM $30 \mathrm{~m}$ single dish telescope and Plateau de Bure Interferometer (PdBI).

\section{Data and Results}

\subsection{CR ionization rate}

[9] sampled five positions toward this interacting regions using the IRAM 30m single dish telescope (Fig. 3 left) and they derived the abundance ratio $\mathrm{DCO}^{+} / \mathrm{HCO}^{+}$in these positions. In the direction of the position W51C-E, the CR ionization rate is estimated to be $\sim 10^{-15} \mathrm{~s}^{-1}$, about 100 times larger than in standard molecular clouds.

Promptly after the publication of this measurement, NIR observation of the same region by [2] revealed the presence of the protostar at the edge of the beam of the $\mathrm{DCO}^{+}$and $\mathrm{HCO}^{+}$observations (Fig 3 right). If [9] observations were contaminated by emission toward the protostar, this could alter the interpretation of their results since the beam-averaged measured ionization rate may not be related to the $\mathrm{CR}$. Therefore we carried out high spatial resolution observations of this region with the PdBI in order to verify if the $\mathrm{DCO}^{+}$and $\mathrm{HCO}^{+}$emission are linked to the protostar [10]. Details on the observations and calibration parameters can be found in [10]. The final naturalweighted data cubes have a beam size of 3.8 " $\times 3.4$ " at $2 \mathrm{~mm}$ and 7.4 " $\times 4.4$ " at $3 \mathrm{~mm}$. The rms is of about $3.1 \mathrm{mJy}^{-1}$ beam ${ }^{-1}$ in $1.3 \mathrm{~km} \mathrm{~s}^{-1}$ channel width at $2 \mathrm{~mm}$ and $4.8 \mathrm{mJy}$ beam ${ }^{-1}$ in $1.1 \mathrm{~km} \mathrm{~s}^{-1}$ channel width at $3 \mathrm{~mm}$. 

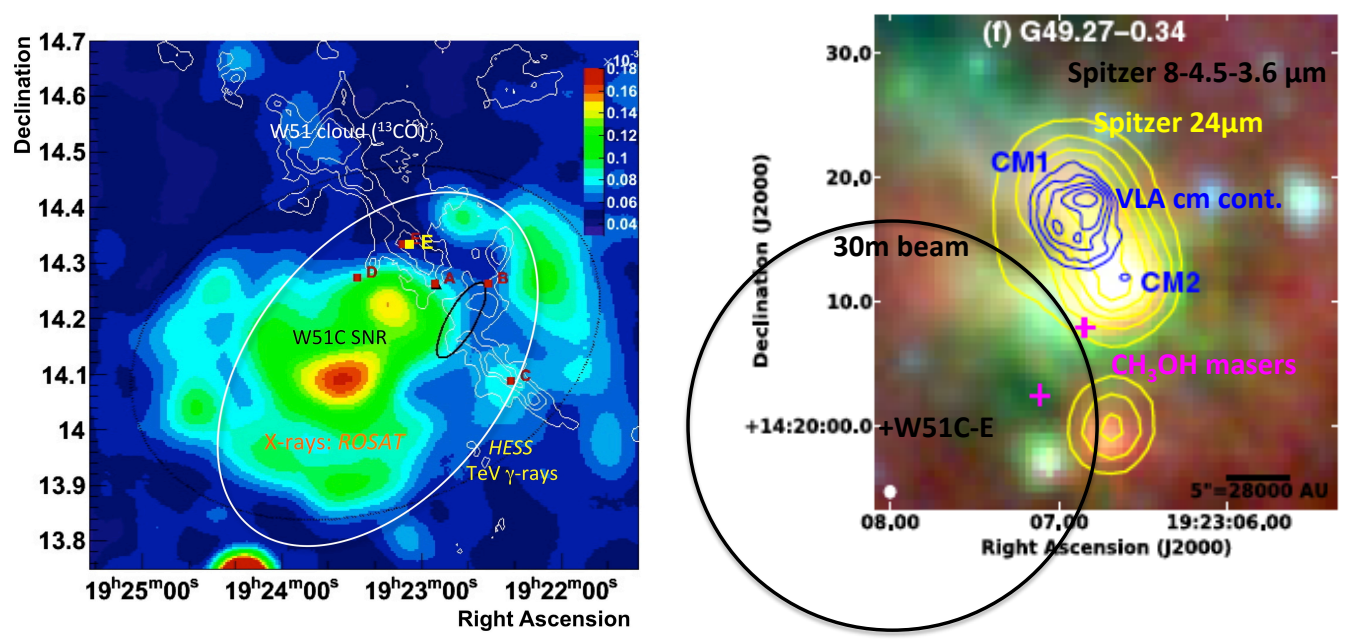

Figure 3: Left: positions observed with the 30m telescope (red squares from A to E) overlaid on the Rosat $X$-ray map (color map). Right: [2] figure: Spitzer NIR emission (color map and yellow contours) and radio continuum emission in blue contours. $\mathrm{CH}_{3} \mathrm{OH}$ masers are shown as the pink crosses. The black cross shows the position of the $30 \mathrm{~m}$ observations discussed in the text, and the black circle represents the $30 \mathrm{~m}$ beam size at $3 \mathrm{~mm}(28$ ").

Figure 4 is showing the spatial distribution of the $\mathrm{DCO}^{+}$and $\mathrm{H}^{13} \mathrm{CO}^{+}$emission lines in the field of view. No compact source is found within the region covered by the IRAM $30 \mathrm{~m}$ observation of W51C-E by [9]. Therefore no emission associated with the nearby protostar is contaminating their measurements, which supports the conclusion by these authors that the entire region probed by the $30 \mathrm{~m}$ beam has an enhanced CR ionization flux.

\section{$\mathrm{DCO}^{+}$}

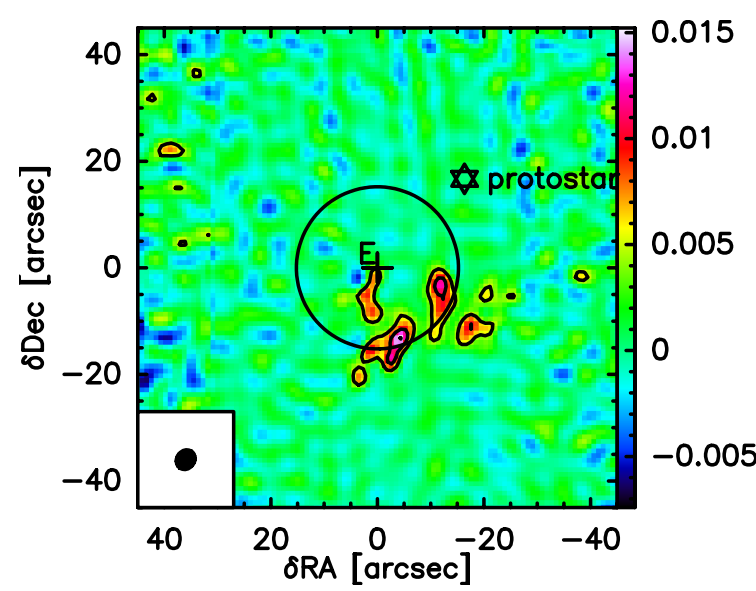

$\mathrm{H}^{13} \mathrm{CO}^{+}$

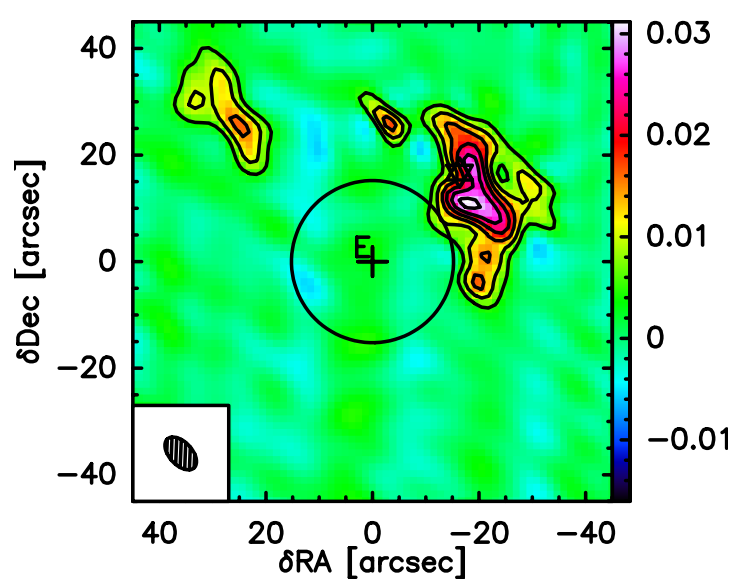

Figure 4: $\mathrm{DCO}^{+}$(left) and $\mathrm{H}^{13} \mathrm{CO}^{+}$(right) integrated intensity in the PdBI field of view. The central cross shows the phase center of the PdBI observation, which is also the position W51C-E of the 30m observations showing enhanced ionization rate. The black circle represents the 30m beam of 28 " at 3mm. The protostar is shown as the black star, north east of the phase center. 


\section{2 $\mathrm{SiO}(2-1)$ emission}

Figure 5 shows the $\mathrm{PdBI} \mathrm{SiO}(2-1)$ integrated map [10]. $\mathrm{SiO}$ emission is concentrated in two regions, north and south of the protostar. The northern region splits into 3 clumps, aligned in the west-east direction over about $1.2 \mathrm{pc}$. The southern structure is weaker and slits into 2 clumps elongated of about $0.5 \mathrm{pc}$. These $\mathrm{SiO}$ clumps are barely resolved at our spatial resolution. The channel maps are shown in figure 6 at a $1.08 \mathrm{~km} \mathrm{~s}^{-1}$ velocity resolution. The emission is present between -6.5 and $+6.5 \mathrm{~km} \mathrm{~s}^{-1}$ around the systemic velocity $\left(67 \mathrm{~km} \mathrm{~s}^{-1}\right)$ and has almost a constant intensity in both north and south $\mathrm{SiO}$ structures. Moreover no velocity gradient, nor structure apparently associated with the protostar is evident.

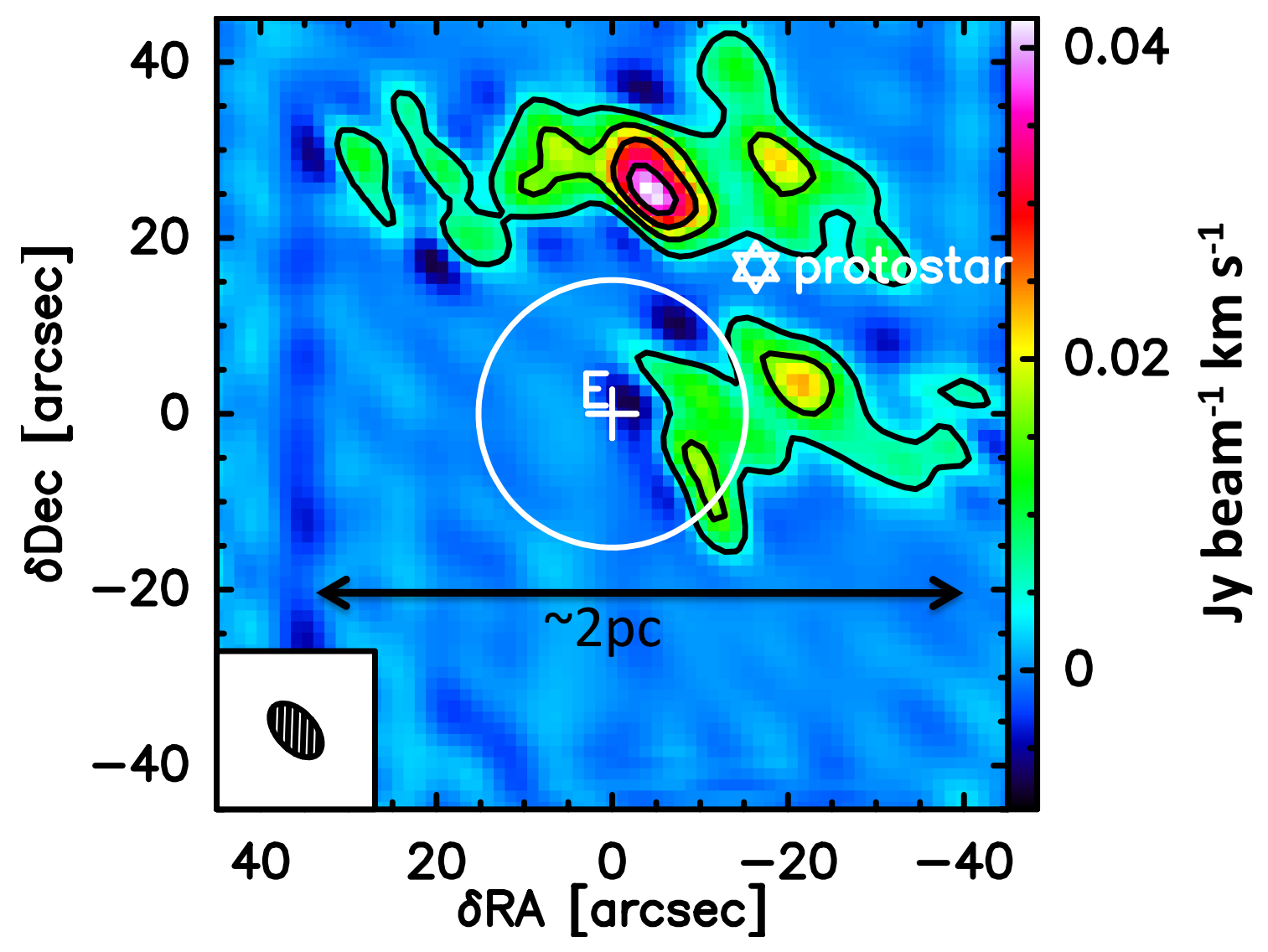

Figure 5: PdBI SiO(2-1) integrated emission. The contours are from 0.005 to 0.05 in steps of $0.01 \mathrm{Jy}$ beam $^{-1} \mathrm{~km} \mathrm{~s}^{-1}$. The white circle represents the primary beam of the IRAM 30m telescope at $3 \mathrm{~mm}(28$ ”), centered on the W51C-E position (white cross). The white star marks the position of the protostar.

\section{Conclusions}

Our PdBI observations showed SiO emission associated with the W51C-E region, where an enhanced $\mathrm{CR}$ ionization rate has been measured. $\mathrm{SiO}$ is commonly used to trace molecular shocks [11] and although our observations do not allow us to give an estimate of the abundance or the physical conditions of the gas, there is no doubt that the observed $\mathrm{SiO}$ emission is tracing shocks 


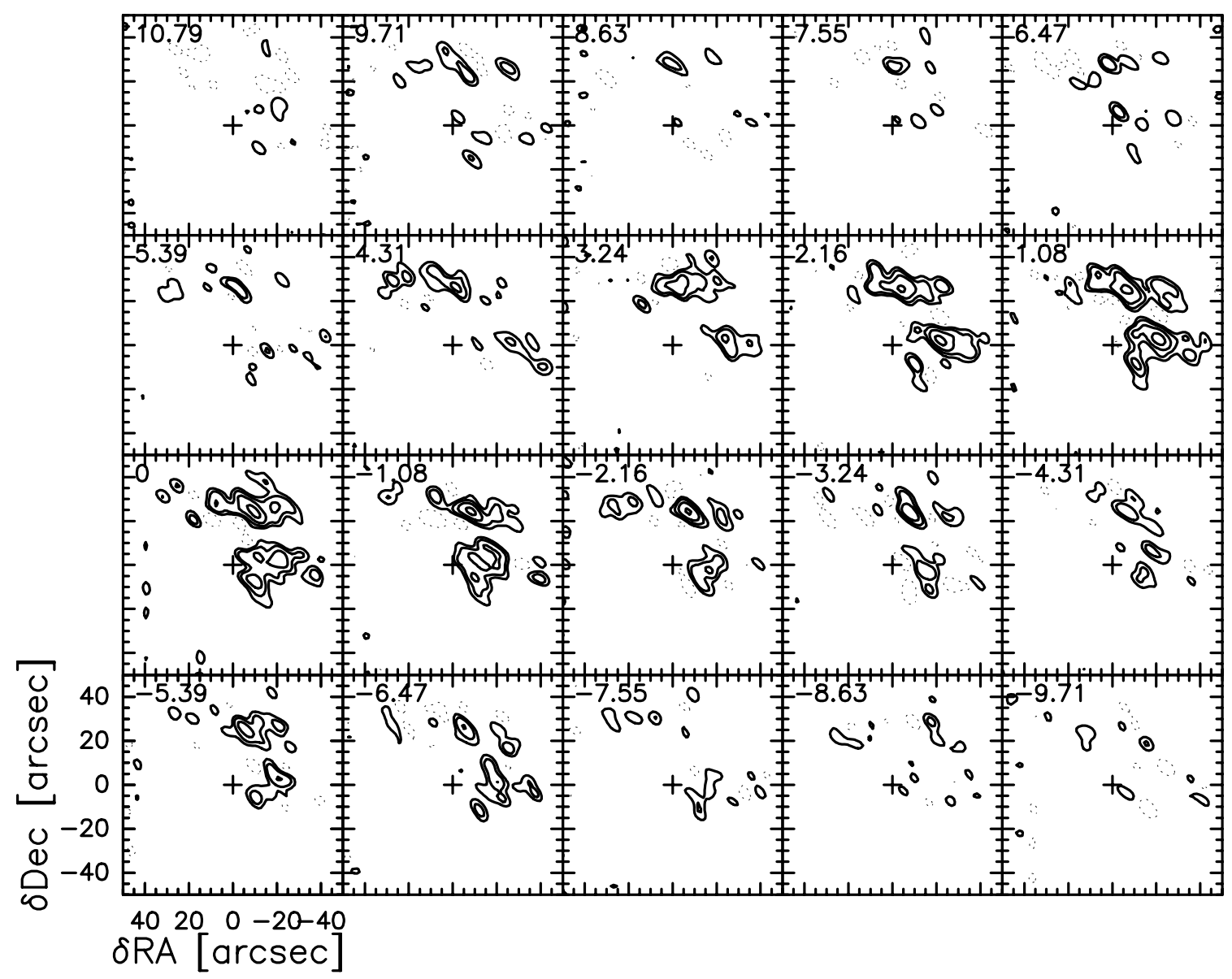

Figure 6: Channel maps of the naturally weighted $\mathrm{SiO}(2-1)$ emission. The velocity resolution is $1.08 \mathrm{~km} \mathrm{~s}^{-1}$ and the channels are shown between -9.7 and $+10.8 \mathrm{~km} \mathrm{~s}^{-1}$ around the LSR velocity $\left(67 \mathrm{~km} \mathrm{~s}^{-1}\right)$. Contours

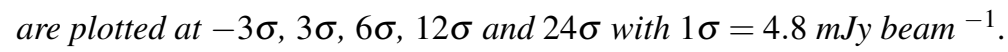

in the molecular gas. What is the origin of this shock? The first possibility is that it is powered by a potential outflow from the nearby protostar. However, no spatial shift between the blue and red shifted emission parts is seen on Fig. 6, and no outflow associated with this protostar was detected by previous studies [12], [2].

The second explanation is that the detected shock is linked to the SNR primary shock that may have created the large flux of CR probed by the enhanced ionization in W51C-E. From thermal $\mathrm{X}$-ray emission, [3] derived a forward shock velocity of about $500 \mathrm{~km} \mathrm{~s}^{-1}$ and a dynamical age of about $3 \times 10^{4} \mathrm{yr}$ for the SNR W51C. If a clump of dense gas obstructs the passage of this primary shock, its propagation stalls and a complex system of shocks develops in and around the clump [13], as shown schematically in figure 7. The localised $\mathrm{SiO}$ emission detected in W51C-E can then arise from such a clump, overrun by the SNR. Given an average overdensity of the cloud of the order of 40 and a primary shock speed of $500 \mathrm{~km} \mathrm{~s}^{-1}$, this would give a typical transmitted shock speed of about $25 \mathrm{~km} \mathrm{~s}^{-1}$, enabling grain sputtering and explaining the $\mathrm{SiO}$ emission [14].

We conclude then that the W51C-E region is downstream of the SNR primary shock, exactly where accelerated CRs would accumulate and fuse the enhanced ionization measured in this region. 


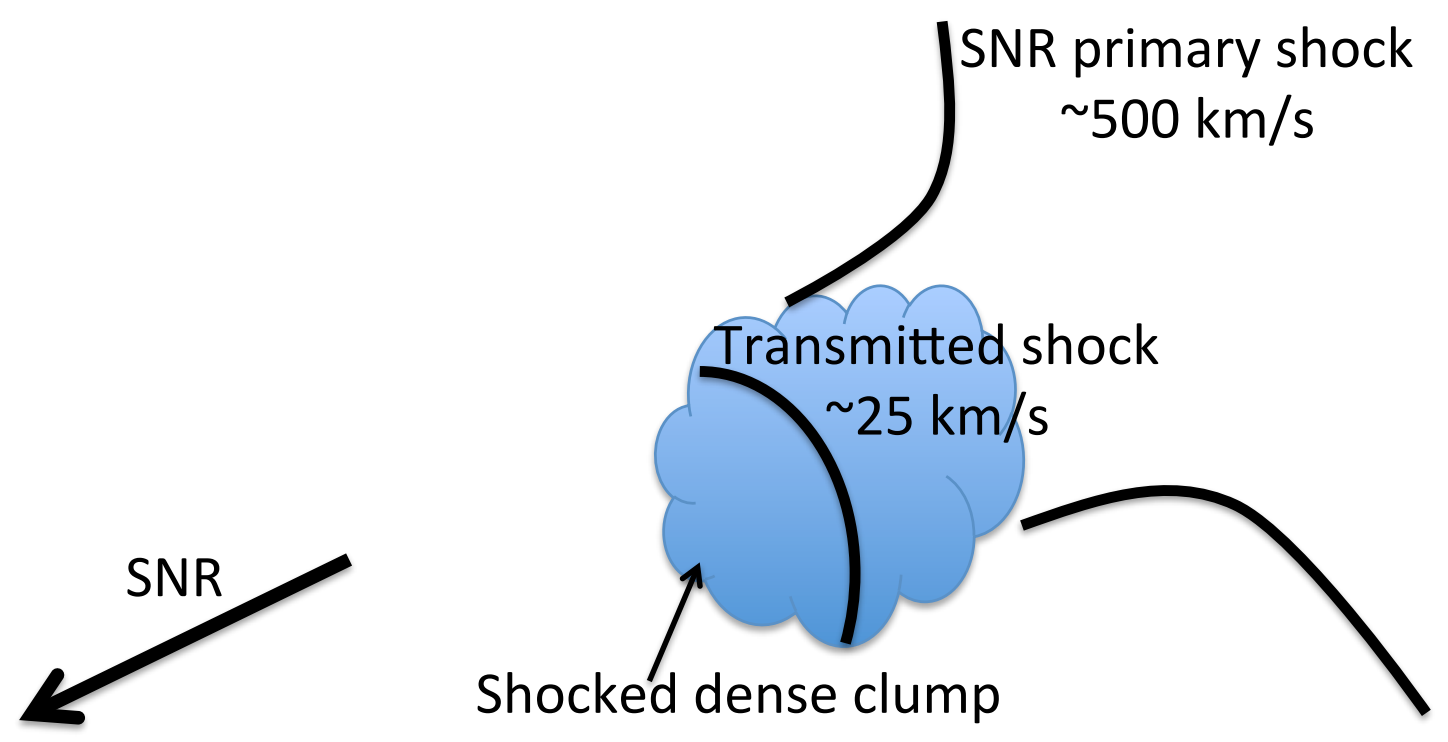

Figure 7: Schematic view of the W51C-E region, showing the transmitting shock developing in the dense molecular medium after the passage of the SNR primary shock

\section{References}

[1] Padovani, M.; Galli, D. \& Glassgold, A. E. 2009, A\&A 501, 619

[2] Cyganowski, C. J.; Brogan, C. L.; Hunter, T. R. \& Churchwell, E.. 2011, ApJ 743, 56

[3] Koo, B-C.; Kim, K-T. \& Seward, F.D. 1995, ApJ 447, 211

[4] Feinstein, F. et al. 2009, AIPC 1112 54F

[5] Abdo, A. A. et al. 2009, ApJ 706, L1

[6] Aleksić, J. et al. 2012, A\&A 541, 13

[7] Jackson, J. M. et al. 2006, ApJS 163, 145

[8] Guélin, M.; Langer, W.D.; Snell, R.L. \& Wootten, H.A. 1977, ApJ 217, L165

[9] Ceccarelli, C.; Hily-Blant, P.; Montemerle, T.; Dubus, G.; Gallant, Y. \& Fiasson, A. 2011, ApJL 740, L4

[10] Dumas G.. et al. 2014, ApJL 786, L24

[11] Bachiller, R. 1996, ARA\&A 35, 11

[12] de Buizer, J. M. \& Vacca, W. D. 2010, ApJ 140, 196

[13] Inoue, T.; Yamazaki, R.; Inutsuka, S.-I. \& Fikui, Y. 2012, ApJ 744, 71

[14] Gusdorf, A.; Pineau des Forets, G.; Cabrit, S. \& Flower, D. R. 2008, A\&A 490, 695 